\title{
A Statistical Comparative Study OF SOME SORTING ALGORITHMS
}

\author{
Anchala Kumari ${ }^{1}$, Niraj Kumar Singh ${ }^{2 *}$ and Soubhik Chakraborty ${ }^{3}$ \\ ${ }^{1}$ Department of Statistics, Patna University, Patna \\ ${ }^{2}$ Department of Computer Science \& Engineering, BIT Mesra, Ranchi \\ ${ }^{3}$ Department of Mathematics, BIT Mesra, Ranchi
}

\begin{abstract}
This research paper is a statistical comparative study of a few average case asymptotically optimal sorting algorithms namely, Quick sort, Heap sort and $K$ - sort. The three sorting algorithms all with the same average case complexity have been compared by obtaining the corresponding statistical bounds while subjecting these procedures over the randomly generated data from some standard discrete and continuous probability distributions such as Binomial distribution, Uniform discrete and continuous distribution and Poisson distribution. The statistical analysis is well supplemented by the parameterized complexity analysis.
\end{abstract}

\section{KEYWORDS}

Parameterized complexity, Statistical bound, Empirical-O, Computer experiment.

\section{INTRODUCTION}

Quick sort and Heap sort are the two standard sorting techniques used in literature for sorting large data sets. These two methods exhibit the very same average case complexity of $O\left(\operatorname{Nlog}_{2} N\right)$, where $N$ is the size of input to be sorted. Quick sort, possibly, is the best choice for sorting random data. The sequential access nature of this algorithm keeps the associated constant terms small and hence resulting in an efficient choice among the algorithms with similar asymptotic class. The worst case complexity of quick sort is that of $O\left(N^{2}\right)$ while the heap sort in worst case exhibit the same $\left(\mathrm{Nlog}_{2} N\right)$ complexity. The $\Theta\left(\mathrm{Nlog}_{2} N\right)$ tight complexity of heap sort gives it an edge over much used quick sort for use in stringent real time systems. New-sort, an improved version of Quick sort which introduced by Sundararajan and Chakarborty [1] also confirms to the same average and worst case complexity as that of Quick sort ,i.e., $O\left(N \log _{2} N\right)$ and $O\left(N^{2}\right)$ respectively. But this New sort technique uses an auxiliary array, increasing the space complexity thereby. A further improvement over the New sort was made by removing the concept of auxiliary array from it and this sorting algorithm was named as K-sort [2]. Interestingly, for typical inputs, on average the $K$-sort also consumes $O\left(\mathrm{Nlog}_{2} N\right)$ time complexity. In a recent research paper Singh et al. [3] explores the quick sort algorithm in detail, where it discusses various interesting patterns obtained for runtime complexity data.

In this paper the three sorting algorithms all with the same average case complexity have been compared by obtaining the corresponding statistical bounds while subjecting these procedures over the randomly generated data from some standard discrete and continuous probability distributions such as Binomial distribution, Uniform discrete and continuous distribution and Poisson distribution. 
International Journal in Foundations of Computer Science \& Technology (IJFCST), Vol.5, No.4, July 2015

\section{ASYMPTOTIC ANALYSIS}

Asymptotic analysis of Quick sort: The average and the best case recurrence of quick sort is $T(N)$ $=2 T(N / 2)+\theta(N), T(1)=0$. This upon solving yields a running time of $O\left(N \log _{2} N\right)$. The worst case recurrence is $T(N)=T(N-1)+\Theta(N), T(1)=0$, which results in $O\left(N^{2}\right)$ complexity of quick sort algorithm.

Asymptotic analysis of Heap sort: The best and worst case complexity of heap sort belong to $\Theta\left(\mathrm{Nlog}_{2} \mathrm{~N}\right)$ complexity.

Asymptotic analysis of $\boldsymbol{K}$-sort: Due to its peculiar similarity the asymptotic time complexities of $K$-sort is similar to that of quick sort.

\section{EMPERICAL-O ANALYSIS}

Empirical-O is an empirical estimate of the statistical bound over a finite range, obtained by supplying numerical values to the weights which emerge from computer experiment. A computer experiment being defined as a series of runs of a code for various inputs and is called deterministic if it gives identical outputs if the code is re run for the same input.

Statistical bound (non probabilistic): If $W_{i j}$ is the weight of (a computing) operation of type $\mathrm{i}$ in the $j^{\text {th }}$ repetition (generally time is taken as a weight) and $y$ is a "stochastic realization of the deterministic $T=\sum 1 . W_{i j}$ where we count one for each operation repetition irrespective of the type, the statistical bound of the algorithm is the asymptotic least upper bound of $y$ expressed as a function of $N, N$ being the algorithm's input size. $T$ is realised for a fixed input while $y$ is expressed for a fixed size of the input. It is important to know $y$ becomes stochastic for those algorithms where fixing size does not fix all the computing operations. Sorting algorithm fall in this category

Now we perform the empirical analysis of the results obtained by applying the specified algorithms over the input data generated from the probability distributions mentioned earlier. The codes were written in Dev C++ 5.8.2 and analysis was performed using Minitab statistical Package.

The response (CPU time to run the code), the mean time in seconds is given in the tables 1-4 and relative performance plots are presented in figures 1-4. Average case analysis is performed directly on program run time to estimate the weight based statistical bound over a finite range by running computer experiments [4][5]. This estimate is called empirical-O[6]][7]. Time of an operation is taken as weight. Weighing permits collective consideration of all operations into a conceptual bound which we call a statistical bound in order to distinguish it from the count based mathematical bounds that are operation specific.

Sample size: The various inputs vary from a size of 1-10 lac which may be considered as reasonably large for practical data set.

\section{RELATIVE PERFORMANCE ANALYSIS OF DIFFERENT ALGORITHMS}

\subsection{Discrete Uniform Distribution}

Discrete Uniform distribution is characterised by single parameter $k$. In this experiment $k$ has been fixed at 1000 . 
International Journal in Foundations of Computer Science \& Technology (IJFCST), Vol.5, No.4, July 2015

Table 1. Data for discrete uniform distribution

\begin{tabular}{|l|l|l|l|}
\hline$N$ & $\begin{array}{l}\text { Heap sort } \\
(H S)\end{array}$ & $\begin{array}{l}\text { K-Sort } \\
(\text { KS })\end{array}$ & $\begin{array}{l}\text { Quick Sort } \\
(Q S)\end{array}$ \\
\hline 100000 & .0528 & .0526 & .03040 \\
\hline 200000 & .0998 & .1842 & .0966 \\
\hline 300000 & .1778 & .4032 & .1904 \\
\hline 400000 & .2436 & .6340 & .3096 \\
\hline 500000 & .2878 & .9424 & .4750 \\
\hline 600000 & .3472 & 1.3376 & .6594 \\
\hline 700000 & .4028 & 1.775 & .860 \\
\hline 800000 & .4622 & 2.886 & 1.092 \\
\hline 900000 & .5216 & 2.9168 & 1.301 \\
\hline 1000000 & .5828 & 3.5912 & 1.744 \\
\hline
\end{tabular}

Some interesting results are seemed to emerge from the above table. For $N<200000$, quick sort gives better performance as heap sort compared to other two algorithms but for $N>=30000$, the heap sort outperforms the quick sort. The reason for ill performance of Quick sort may is due to increase in number of ties in data set as $N$ increases. As for as the $K$-sort is considered it gives the worst performance at this particular value of $\boldsymbol{k}$.

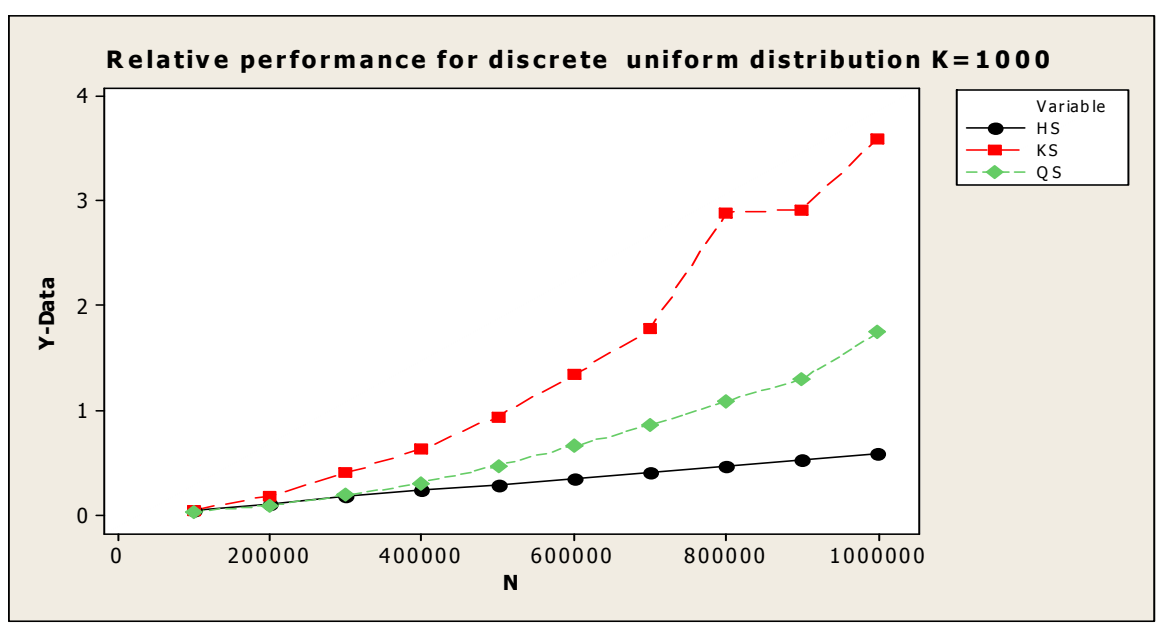

Figure 1. Relative plots of quick heap and K-sort algorithms $(\mathrm{k}=1000)$

For Discrete Uniform inputs in average case Quick sort, and $K$-Sort exhibit a time complexity of $O_{e m p}\left(N^{2}\right)$ whereas that of heap sort it is $O_{e m p}\left(N \log _{2} N\right)$ [2].

\subsection{Binomial Distribution}

The Binomial distribution has two parameters $m$ and $p, m$ being the number of independent Bernoulli trials and $p$ the probability of success in each trial. The mean time given in table 2 was obtained by varying $\mathrm{N}$ between 100000 to 1000000 and fixing $m$ and $p$ at 1000 and 0.5 respectively and making several runs for the same input. 
International Journal in Foundations of Computer Science \& Technology (IJFCST), Vol.5, No.4, July 2015

Table 2. Data for Binomial distribution

\begin{tabular}{|c|c|c|c|}
\hline$N$ & $\begin{array}{c}\text { Heap sort } \\
(H S)\end{array}$ & $\begin{array}{c}\text { K-sort } \\
(\text { KS })\end{array}$ & $\begin{array}{c}\text { Quick } \\
\text { sort } \\
(Q S)\end{array}$ \\
\hline 100000 & .4998 & .6096 & .2872 \\
\hline 200000 & 1.0450 & 4.0198 & 1.1166 \\
\hline 300000 & 1.6344 & 5.3716 & 2.46360 \\
\hline 400000 & 2.24000 & 9.9072 & 4.3850 \\
\hline 500000 & 2.8534 & 15.02379 & 6.8992 \\
\hline 600000 & 3.4602 & 22.8832 & 9.8526 \\
\hline 700000 & 4.0792 & 30.51688 & 13.5338 \\
\hline 800000 & 4.74800 & 41.0869 & 17.66879 \\
\hline 900000 & 5.3766 & 49.628720 & 22.0784 \\
\hline 1000000 & 5.9798 & 62.4471 & 22.2676 \\
\hline
\end{tabular}

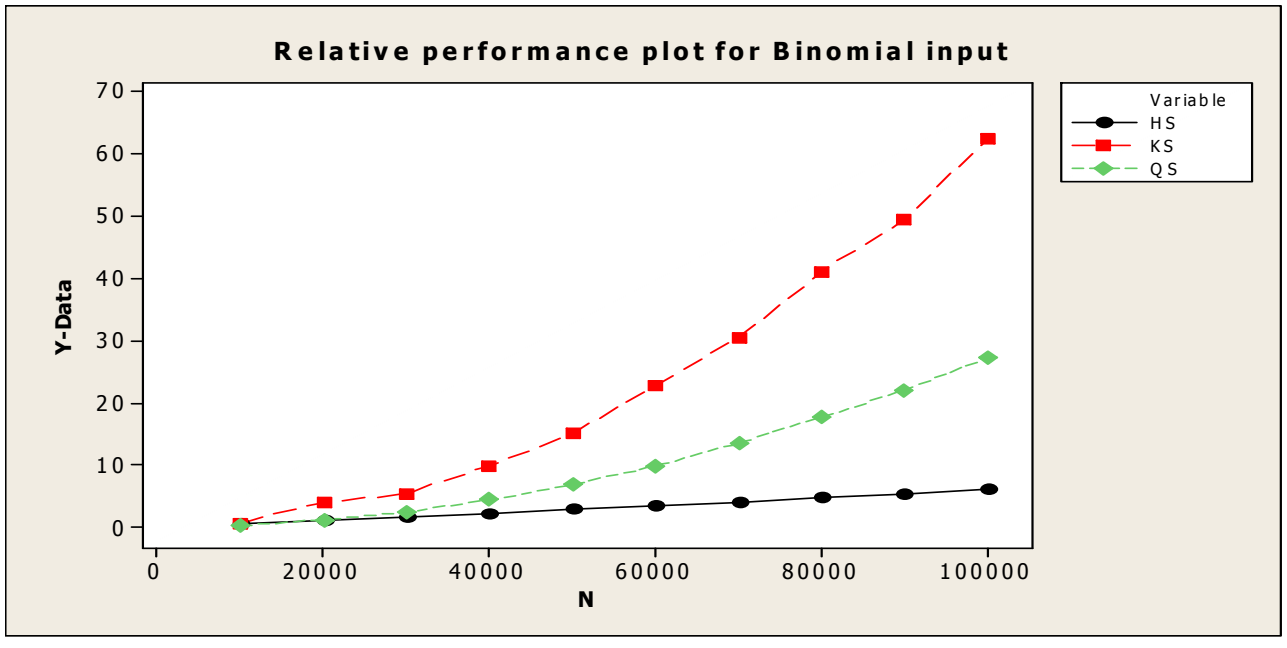

Figure 2. Relative plots of quick heap and K-sort algorithms $(\mathrm{m}=1000$ and $\mathrm{p}=0.5)$

The input data table (2) and relative performance plot (figure 2) supports the fact that on the average $K$-sort consumes more time as compared to other two algorithms for sorting array of same size. However for Binomial inputs quick sort and $K$-sort both confirms to $O_{\text {emp }}\left(N^{2}\right)$, whereas heap sort has $\mathrm{O}\left(\mathrm{Nlog}_{2} \mathrm{~N}\right)$ complexity.

\subsection{Poisson Distribution}

The Poisson distribution depends on the parameter $\lambda$. Lambda (which is both the mean and the variance) should not be large as this is the distribution of rare events. While performing the empirical analysis with Poisson inputs, its parameter, $\lambda$ is fixed at 5.0 
International Journal in Foundations of Computer Science \& Technology (IJFCST), Vol.5, No.4, July 2015

Table 3. Data for Poisson Input

\begin{tabular}{|l|l|l|l|}
\hline$N$ & $\begin{array}{l}\text { Heap sort } \\
(H S)\end{array}$ & $\begin{array}{l}\text { K-sort } \\
(\text { KS })\end{array}$ & $\begin{array}{l}\text { Quick } \\
\text { sort } \\
(\text { QS })\end{array}$ \\
\hline 20000 & .015 & .1778 & .0964 \\
\hline 40000 & .019 & .7158 & .3190 \\
\hline 60000 & .0342 & 1.7450 & .7250 \\
\hline 80000 & .047 & 2.8082 & 1.2696 \\
\hline 100000 & .0636 & 4.5356 & 1.9696 \\
\hline 120000 & .0716 & 6.320 & 2.8502 \\
\hline 140000 & .0818 & 8.5972 & 3.8226 \\
\hline 160000 & .094 & 11.2604 & 4.979 \\
\hline 18000 & .110 & 14.736 & 6.3406 \\
\hline 200000 & .125 & 18.0549 & 7.8044 \\
\hline
\end{tabular}

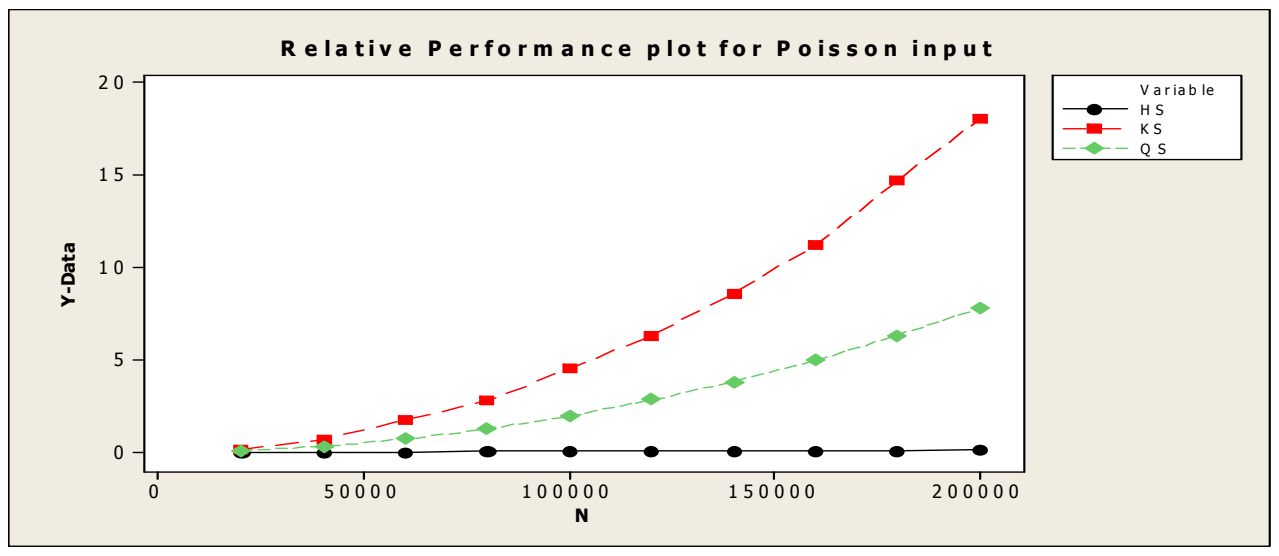

Figure 3. Relative plots of quick heap and K-sort algorithms $(\lambda=5.0)$

For Poisson inputs, heap sort gives the good performance as compared to other two algorithms. Quick sort and $K$-sort both have $O_{e m p}\left(N^{2}\right)$ complexity whereas if we have a look on the three graph (figure 3), it is obvious that heap sort again confirms to $O_{\text {emp }}\left(\operatorname{Nlog}_{2} N\right)$ complexity,

\subsection{Uniform Continuous Distribution}

Table 4 gives the mean execution time for the data simulated from uniform continuous distribution $[0, \theta]$.

Table 4. Data for Uniform Continuous Distribution

\begin{tabular}{|l|l|l|l|}
\hline$N$ & $\begin{array}{l}\text { Heap sort } \\
(H S)\end{array}$ & $\begin{array}{l}\text { K-sort } \\
(\text { KS })\end{array}$ & $\begin{array}{l}\text { Quick } \\
\text { sort } \\
(\text { QS })\end{array}$ \\
\hline 100000 & .055 & .0310 & .0340 \\
\hline 200000 & .11277 & .0748 & .062 \\
\hline 300000 & .1780 & .1092 & .0872 \\
\hline
\end{tabular}


International Journal in Foundations of Computer Science \& Technology (IJFCST), Vol.5, No.4, July 2015

\begin{tabular}{|l|l|l|l|}
\hline 400000 & .2400 & .1468 & .1064 \\
\hline 500000 & .3150 & .2002 & .1312 \\
\hline 600000 & .3842 & .2624 & .159 \\
\hline 700000 & .4533 & .3092 & .1908 \\
\hline 800000 & .5188 & .3672 & .219 \\
\hline 900000 & .902 & .425 & .2534 \\
\hline 10000 & 1.0154 & .4750 & .2842 \\
\hline
\end{tabular}

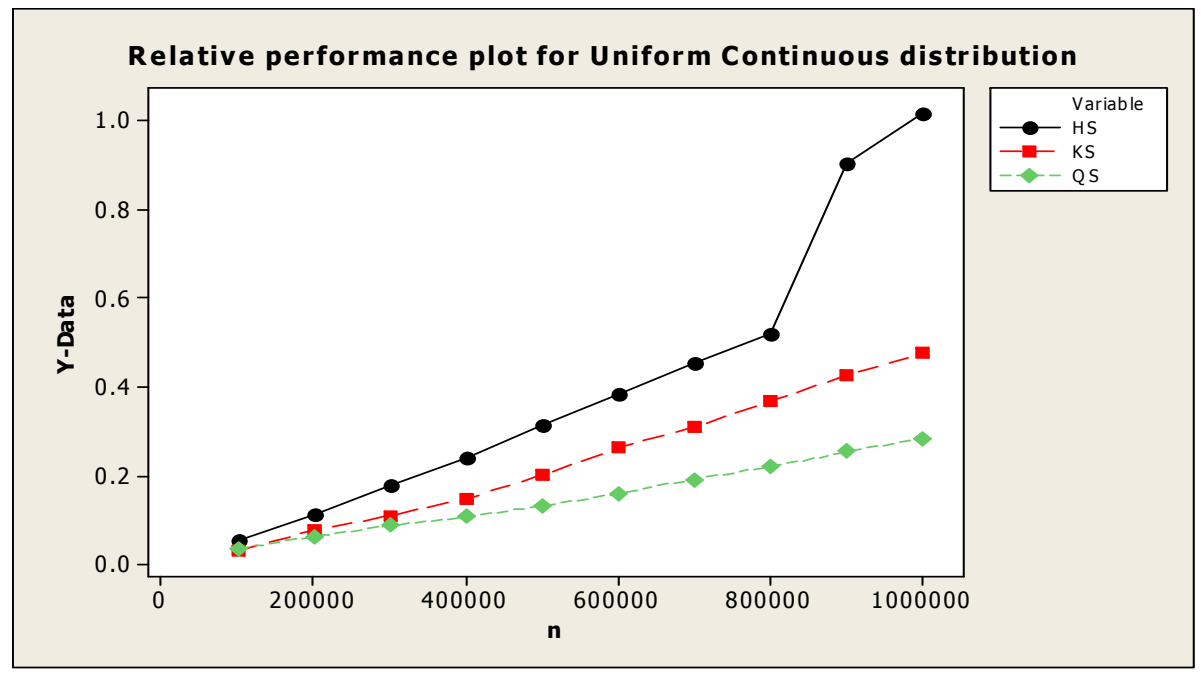

Figure 4. Relative plots of quick heap and K-sort algorithms [0-1]

Here the scenario has just changed. Quick sort outperforms the two algorithms in its performance. The $K$-sort for all values of $N$ in selected range out performance the heap algorithm . However all the three algorithms suggest $O_{e m p}\left(N \log _{2} N\right)$ complexity.

\section{STATISTICAL ANALYSIS OF DIFFERENT ALGORITHMS FOR DISCRETE DISTRIBUTIONS}

In this section we test the hypothesis whether the average performance of the algorithms for different input distributions is same or not. For this we apply two way analysis of variance. A value of $p=0.358$ greater than 0.05 as shown in table 5 , is indicative of the fact that as for as their average performance is concerned there is no reason to differentiate between the three.

Table 5. Results of Two Way ANOVA

$\begin{array}{lrrrrr}\text { Source } & \text { DF } & \text { SS } & \text { MS } & \text { F } & \text { P } \\ \text { PRODIS } & 3 & 163.354 & 54.4514 & 2.52 & 0.155 \\ \text { SORTALG } & 2 & 52.955 & 26.4776 & 1.23 & 0.358 \\ \text { Error } & 6 & 129.602 & 21.6003 & & \\ \text { Total } & & 11345.911 & & & \end{array}$




\subsection{Parametric complexity}

Parametric complexity is one of the important criterions for selection of an algorithm among the several algorithms, since besides the size of input, parameters of input distribution has direct effect on execution time of an algorithm. We have examined the parameterized complexity of the three algorithms for binomial inputs, the reason being that distribution has two parameters . A $3^{2}$ factorial experiment with three repeated set of data elements at same combination of level of factors $m$ and $p$ has been employed. The results given in table 6 below reveal some interesting findings:

Table 6. Parameterized Complexity of heap sort, k-sort and quick sort

\begin{tabular}{|c|c|c|c|c|c|c|c|}
\hline \multirow[t]{2}{*}{ sources } & \multirow[b]{2}{*}{$\mathrm{df}$} & \multicolumn{2}{|l|}{$\begin{array}{l}\text { Heap } \\
\text { sort } \\
\text { (HS) }\end{array}$} & \multicolumn{2}{|l|}{$\begin{array}{l}\text { K-sort } \\
\text { (KS) }\end{array}$} & \multicolumn{2}{|l|}{$\begin{array}{l}\text { Quick } \\
\text { sort } \\
\text { (QS) }\end{array}$} \\
\hline & & $\mathrm{F}$ & $\mathrm{P}$ & $\mathrm{F}$ & $\mathrm{P}$ & $\mathrm{F}$ & $\mathrm{P}$ \\
\hline$\underline{\mathrm{M}}$ & $\underline{2}$ & $\underline{0.13}$ & .876 & $\underline{3.85}$ & .04 & $\underline{1.11}$ & .351 \\
\hline$\underline{P}$ & $\underline{2}$ & $\underline{0.15}$ & .866 & 37.37 & $\underline{0.00}$ & $\frac{26.1}{8}$ & .000 \\
\hline$\underline{\mathrm{MP}}$ & $\underline{4}$ & $\underline{0.18}$ & .948 & 1.62 & .213 & $\underline{0.31}$ & .867 \\
\hline
\end{tabular}

The $F$ and $P$ values revealed that in case of Heap Sort the two parameters neither singularly nor jointly has any effect on sorting time while in case of $K$-sort though both the factors have independent effects on the complexity, probability of success being highly significant. While applying the quick sort algorithm, the number of trials $(m)$ shows highly non significant effect while the probability of success $p$ delivers significantly high effect on complexity .Thus the proper selection of input parameters can have rewarding effect on reducing the complexity of an algorithm.. For different values of $p$, the average execution time is given in the table 7 below

Table 7. optimal value of $\mathrm{p} m=5000, \mathrm{n}=50000$

\begin{tabular}{|c|c|c|}
\hline $\mathrm{P}$ & K-sort & Quick sort \\
\hline 0.1 & .188 & .056 \\
\hline 0.2 & .144 & .047 \\
\hline 0.3 & .126 & .040 \\
\hline 0.4 & .127 & .044 \\
\hline 0.5 & .115 & .0438 \\
\hline 06 & .117 & .0566 \\
\hline 0.7 & .125 & .063 \\
\hline 0.8 & .1406 &. .072 \\
\hline 0.9 & .183 &. .072 \\
\hline
\end{tabular}

Here we find that in both the cases initially the execution time decreases as the value of $p$ is increased, at $p=.5$, execution time is minimum and then again it goes on increasing. Thus the optimal value of $p$ is .5. But as for the optimal value of $m$ is concerned in case of $k$ sort, as shown in the following table 8, the execution time decreases with increase in the value of $m$ Thus in this case a high value of $m$ is preferable. 
Table 8. optimal value of $\mathrm{m}$ for $\mathrm{K}$ sort $(\mathrm{p}=.5, \mathrm{n}=50000)$

\begin{tabular}{|c|c|}
\hline $\mathrm{M}$ & Ksort \\
\hline 2000 & .174 \\
\hline 3000 & .1456 \\
\hline 4000 & .1275 \\
\hline 5000 & .0782 \\
\hline 6000 & .0704 \\
\hline 7000 & .0666 \\
\hline 8000 & .0644 \\
\hline 9000 & .061 \\
\hline 10000 & .056 \\
\hline
\end{tabular}

\section{CONCLUSIONS}

The three sorting algorithms, heap sort, $K$-sort and quick sort though theoretically deliberating to same complexity $O\left(\operatorname{Nlog}_{2} N\right)$ has been supported by the statistical analysis in section 3 . We have no evidence against rejection of the hypothesis of homogeneity of algorithms as far as their average performance is considered. But unfortunately in worst case quick sort and K-sorts have complexity $O\left(N^{2}\right)$ than heap sort which exhibits a complexity of $O\left(\operatorname{Nlog}_{2} N\right)$ since we have the relation that $O\left(\mathrm{Nlog}_{2} \mathrm{~N}\right)<O\left(\mathrm{~N}^{2}\right)$.

However as far as Empirical-O estimates are considered, quick sort for $\mathrm{N}$ less than 200000 gives better performance for some discrete distributions such as Binomial and Uniform distribution while for $\mathrm{N}>300000$ heap sort is best, $K$-sort for these distributions does not work good. But sorting an array generated randomly (not generated from any standard probability distribution, $K$ sort works good. It can sort an array of size never greater than 7000000 in less time than heap sort [2].

For continuous uniform distribution, quick sort gives the good performance as compared to other two. This result is quite expected as in case of a continuous distribution the probability of getting similar valued elements (ties) is theoretically zero. It is well known through various results [8] that quick sort behaves exceptionally good in such cases. Whereas in the very same scenario heap sort is expected to perform relatively poor (however the time complexity of heap sort remains of the order of $N \log _{2} N$ ) [9].

The different behaviour of the algorithms to input data can be supplemented by the parameterised complexity analysis since the true potential of an algorithm is related to parameters of the input distribution. As far as the parameterised complexity of heap sort is considered, it is in favour of its worst case complexity which is less than the other two algorithms. The reason being obvious as execution time does not depend on the binomial parameters. But in case of quick sort the parameter $p$ has highly significant effect on execution time. Though the two parameters $\mathrm{m}$ and $\mathrm{p}$ have independent effects on complexity in case of $\mathrm{K}$ sort, but we find that parameter $\mathrm{m}$ has a very little effect where as $\mathrm{p}$ has highly significant . In both the cases of K sort and Quick sort, the complexity is minimum at $\mathrm{p}=.5$ but a high value of $\mathrm{p}$ is preferable to reduce the complexity while using the K-sort algorithm. Thus proper selection of input parameters can have rewarding effect on reducing complexity of an algorithm. 
International Journal in Foundations of Computer Science \& Technology (IJFCST), Vol.5, No.4, July 2015

\section{REFERENCES}

[1] Sundararajan, KK \& Chakraborty S, (2007) "A New sorting algorithm" Journal of Applied Ma1thematics and Computation 188, pp 1037-1041.

[2] Sundararajan, KK, Pal, M, Chakarborty ,S., \& Mahanti, NC, (2013) "K-Sort: A New Sorting Algorithm that beats Heap Sort for $\mathrm{n} \leq 70$ lakhs".

[3] Singh, N K, Chakraborty, S, and Mallick, DK, (2014) "A Statistical Peek into Average Case Complexity", IJEDPO, Vol. 4, No 2, Int. J. on Recent Trends in Engineering and Technology 8(1), 64-67.

[4] Fang, KT, Li, R, and Sudjianto, (2000) Design and Modelling of computer Experiments, Taylor \& Francis Group, Boca Raton.

[5] Sacks, J, Welch,W, Mitchel, T, and Wynn, (1989) "Design and Analysis of Computer Experiments", Statistical Science 4.

[6] Chakraborty , S, \& Sourabh, SK, (2010) A Computer Oriented Approach to Algorithmic Complexity, Lambert Academic Publishing.

[7] Chakraborty, S, Modi, N and Panigrahi, S, (2009) "Will the weight based Statistical Bounds Revolutionize the It?", International Journal of Computational Cognition 7(3).

[8] Singh, NK \& Chakraborty, S, (2012) "Smart Sort: A Fast, Efficient and Robust Sorting Algorithm", IJACMS, ISSN 2230-9624, Vol 3, Issue 4, pp 487-493.

[9] Sourabh, SK , and Chakraborty, S, (2009) "Empirical Study on the Robustness of Average Complexity and Parameterized Complexity Measure for Heap sort Algorithm", International Journal of Computational Cognition.Vol.7, No.4.

\section{Authors}

Anchala Kumari is a Professor in the department of statistics, Patna University, India. Her research area includes: Operations research, Design of Experiments and Computer Programming.

Niraj Kumar Singh is a Teaching Cum Research Fellow at department of CSE, BIT Mesra, India. His research interest includes: Design and Analysis of Algorithms and Algorithmic Analysis through Statistical bounds.

Soubhik Chakraborty is a Professor in the department of Mathematics, BIT Mesra, India. His research interest includes: Music Analysis, Algorithmic Complexity and Statistical Computing.
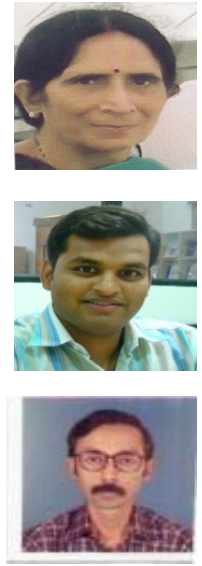